\title{
Funding cuts spark fears about the rise of drug-resistant strains of HIV in Africa
}

Published at www.cmaj.ca on Jan. 7

$\mathrm{T}$ rezar Mnenula sits in a consultation room in Zomba Central Hospital in Malawi, eyeing her HIV-infected patient skeptically. "Why do you think you keep forgetting to take your ARVs [antiretrovirals]?" she asks.

The woman's explanation, that she "forgot" the seven leftover pills in her container, doesn't cut it.

Mnenula, a young clinical officer intern with red-and-black-framed glasses and long, tiny braids, says nothing, waiting for her patient to elaborate. Eventually, she sighs and says, "I'm going to send you for counselling again. Apparently the messages didn't sink in the first time."

It's become an altogether common occurrence in Malawi, and across Africa, driving fears that drug-resistant strains of HIV are emerging because patients don't adhere to their antiretroviral drug therapies, setting back a decade of gains in the battle against AIDS. Drug resistance occurs naturally over years of being on treatment, but can arise in just a few weeks if patients take their drugs intermittently. Antiretroviral therapy suppresses drug replication, but when there are low levels of drugs in the blood, the virus has the opportunity to replicate quickly and mutate into resistant forms.

"In many ways, resistance is a silent problem because we don't have the abilities to measure it," says Adrienne Chan, medical coordinator at Dignitas International, a Canadian organization facilitating AIDS treatment in Malawi. The cost of viral load tests, the best measure for resistance, puts them out of the reach of the majority of patients in sub-Saharan Africa.

Compounding the problem are fears that global funding cuts are leading to shortages of antiretroviral drugs in some nations, and the ensuing possibil-

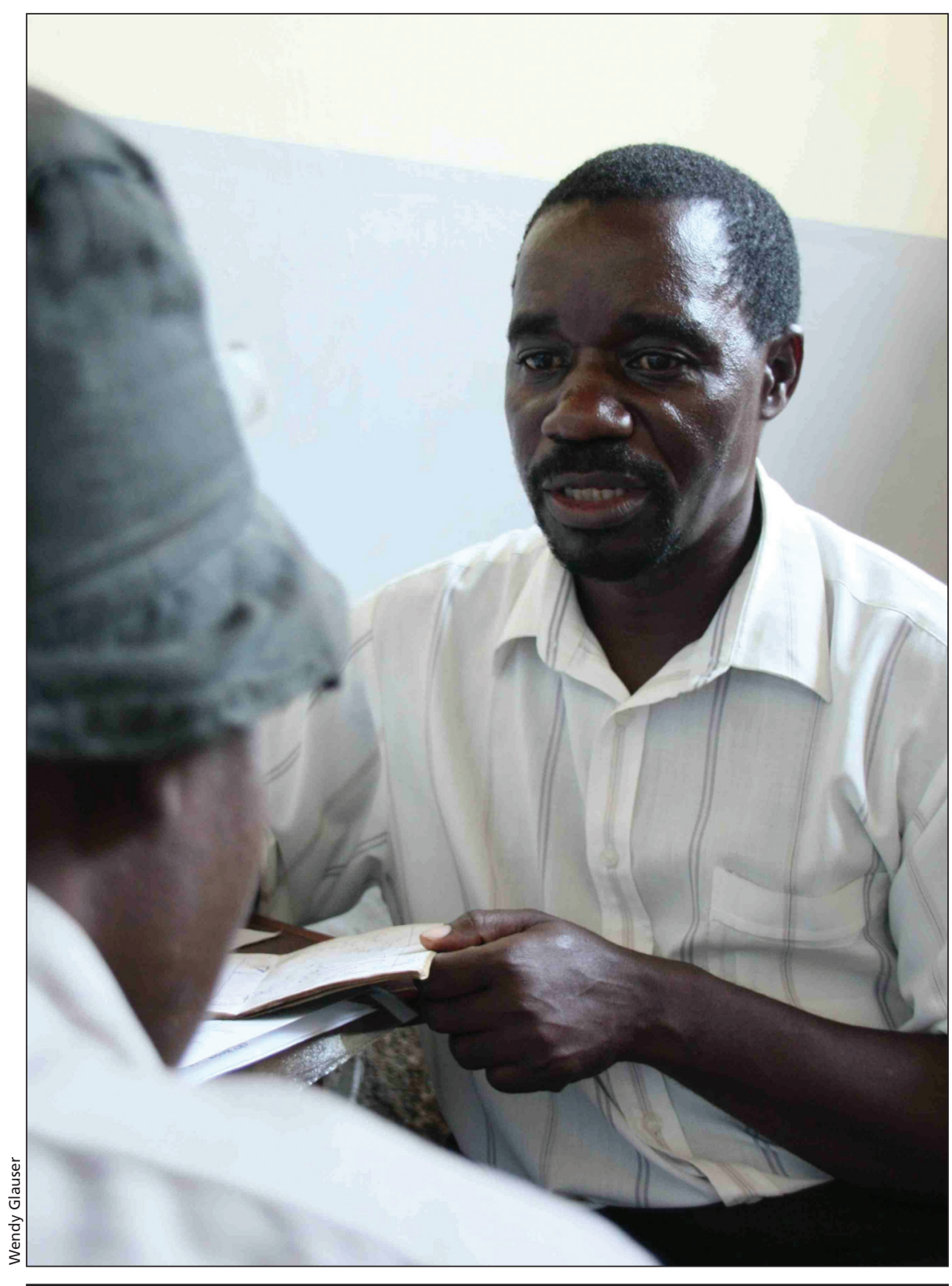

Clinical officer Gabriel Mateyu meets with an HIV-positive patient at the Zomba Central Hospital in Malawi.

ity that the course of treatment of many patients will be interrupted, again raising the likelihood that drug-resistant strains of HIV will emerge.

According to Sharonann Lynch, an HIV/AIDS policy adviser for Médecins Sans Frontières (MSF), "there are already number of stock outs in countries MSF is working in."

The humanitarian agency noted in a recent report, Punishing Success: Early Signs of a Retreat from Commitment to HIV Care and Treatment, that the Global Fund to Fight AIDS, Tuberculosis and 
Malaria funded 35\% fewer proposals in 2009 than in 2008. Meanwhile, after increasing outlays in each of the last five years, the United States President's Emergency Plan for AIDS Relief has capped spending for the next two years.

"The countries have calculated how much they'll need for treatment in five, 10 years and the donors have said how much they're going to give," says Lynch. "There's a significant gap."

Patients share pills when access to antiretrovirals is severely limited, Lynch says, a practice that sends resistance rates soaring. Also, the reintroduction of user fees for AIDS treatment services could mean patients will take drugs when they can afford them, rather than on a routine basis, she adds.

Peter Mugyenyi, executive director of Uganda's Joint Clinical Research Centre, worries the cuts could mean a return to the days when drugs were scarce and resistance was dangerously high. Uganda has an HIV prevalence of $6.5 \%$, and receives most of its treatment support from the US president's fund.

"I want to tell you that I am panicking. Concern is not the word. I am panicking," Mugyenyi says, adding that his centre completed a study in 2003 that tracked 50 patients who'd started on antiretroviral therapy and found that $56 \%$ acknowledged interruptions in their drug regimen and 52\% had drugresistant mutations of the virus (AIDS Research and Human Retroviruses 2004; 20[4]:355-64).

Mugyenyi says the steady supply of antiretrovirals from the president's fund reversed the trend, but Uganda is "now at the gate and about to enter what was happening in the mid-1990s."

Of equal concern is the possibility that the cuts will affect how antiretrovirals are delivered, which might exacerbate the drug resistance problem.

In the Zomba district, patients once walked as many as eight hours to a hospital to get their prescriptions refilled. Since 2006, Dignitas International has been working with local health authorities to decentralize AIDS services to district health centres.

"Before we had ARVs [antiretrovirals] here, the patients would maybe kill a chicken and sell it so that they would have the transport money to get to the hospital," says a clinic nurse. Clinical officer Gabriel Mateyu says many patients would miss their appointments for prescription refills altogether.

Lynch argues that "if you treat people where they live, there's mountains of evidence that you'll have much better success in terms of adherence." But she adds that with less money for training and diagnostic tests, countries may start scaling back the number of facilities offering antiretrovirals.

Dr. Wilford Kirungi, who monitors drug resistance for Uganda's health ministry, says the government will curb the reemergence of resistance by enrolling fewer patients on treatment plans and rigorously ensuring that those receiving treatment adhere to their regimen.

Mugyenyi is furious that Uganda must stop enrolling new patients. Not only will it lead to mass mortality among patients who never get access to treatment, it won't stop the rise of drugresistant HIV, he says. In situations of scarcity, pill sharing goes up; drug shortages are "a recipe for resistance."

$\mathrm{He}$ also takes issue with the rationale behind the US government's decision to focus global health investments on AIDS prevention, rather than treatment, and to redirect money toward improving health systems. "Some kind of experts come here and they advise the US government only that they need to pay attention to such abstracts as mentoring health care providers," he says. "The number one thing is availability of treatment. Any other program, whatever name they call it, will fail." - Wendy Glauser, Zomba, Malawi, and Ben Simon, Kampala, Uganda

DOI:10.1503/cmaj.109-3149 